\title{
Long-read sequencing resolves structural variants in SERPINC1 causing antithrombin deficiency and identifies a complex rearrangement and a retrotransposon insertion not characterized by routine diagnostic methods
}

Belén de la Morena-Barrio ${ }^{1}$, Jonathan Stephens ${ }^{2,3}$, María Eugenia de la Morena-Barrio ${ }^{1}$, Luca Stefanucci ${ }^{2,4,5}$, José Padilla ${ }^{1}$, Antonia Miñano ${ }^{1}$, Nicholas Gleadall ${ }^{2,3}$, Juan Luis García ${ }^{6}$, María Fernanda López-Fernández ${ }^{7}$, Pierre-Emmanuel Morange ${ }^{8}$, Marja K Puurunen ${ }^{9}$, Anetta Undas ${ }^{10}$, Francisco Vidal ${ }^{11}$, NIHR BioResource ${ }^{3}$, F Lucy Raymond ${ }^{3,12}$, Vicente Vicente García ${ }^{1}$, Willem H Ouwehand ${ }^{2,3}$, Javier Corral $^{1,13}$, Alba Sanchis-Juan ${ }^{2,3,13}$

1. Servicio de Hematología y Oncología Médica, Hospital Universitario Morales Meseguer, Centro Regional de Hemodonación, Universidad de Murcia, Instituto Murciano de Investigación Biosanitaria (IMIB-Arrixaca), Centro de Investigación Biomédica en Red de Enfermedades Raras (CIBERER), Murcia, Spain. 2. Department of Haematology, University of Cambridge, NHS Blood and Transplant Centre, Cambridge, CB2 OPT, UK. 3. NIHR BioResource, Cambridge University Hospitals NHS Foundation Trust, Cambridge Biomedical Campus, Cambridge, CB2 OQQ, UK. 4. National Health Service Blood and Transplant (NHSBT), Cambridge Biomedical Campus, Cambridge, CB2 OPT, UK. 5. BHF Centre of Excellence, Division of Cardiovascular Medicine, Addenbrooke's Hospital, Cambridge Biomedical Campus, Cambridge, CB2 0QQ, UK. 6. Servicio de Hematología, Hospital Universitario de Salamanca, Salamanca, Spain. 7. Servicio de Hematología, Complexo Hospitalario Universitario de A Coruña, A Coruña, Spain. 8. Laboratory of Haematology, La Timone Hospital, Marseille, France; C2VN, INRAE, INSERM, Aix-Marseille Université, Marseille, France. 9.National Heart, Lung and Blood Institute's. The Framingham Heart Study, Framingham, MA, US. 10. Institute of Cardiology, Jagiellonian University Medical College and John Paul II Hospital, 80 Prądnicka St, Kraków, Poland. 11. Banc de Sang i Teixits, Barcelona, Spain; Vall d'Hebron Research Institute, Universitat Autònoma de Barcelona (VHIR-UAB), Barcelona, Spain; CIBER de Enfermedades Cardiovasculares, Madrid, Spain. 12. Department of Medical Genetics, University of Cambridge, Cambridge Biomedical Campus, Cambridge, UK. 13. These authors contributed equally to this work.

\begin{abstract}
The identification and characterization of structural variants (SVs) in clinical genetics have remained historically challenging as routine genetic diagnostic techniques have limited ability to evaluate repetitive regions and SVs. Long-read whole-genome sequencing (LR-WGS) has emerged as a powerful approach to resolve SVs. Here, we used LR-WGS to study 19 unrelated cases with type I Antithrombin Deficiency (ATD), the most severe thrombophilia, where routine molecular tests were either negative, ambiguous, or not fully characterized. We developed an analysis workflow to identify disease-associated SVs and resolved 10 cases. For the first time, we identified a germline complex rearrangement involved in ATD previously misclassified as a deletion. Additionally, we provided molecular diagnoses for two unresolved individuals that harbored a novel SINE-VNTR-Alu retroelement insertion that we fully characterized by de novo assembly and confirmed by PCR amplification in all affected relatives. Finally, the nucleotide-level resolution achieved for all the SVs allowed breakpoint analysis, which revealed a replication-based mechanism for most of the cases. Our study underscores the utility of LR-WGS as a complementary diagnostic method to identify, characterize, and unveil the molecular mechanism of formation of diseasecausing SVs, and facilitates decision making about long-term thromboprophylaxis in ATD patients.
\end{abstract}




\section{Main text}

Haploinsufficiency of SERPINC1 (MIM: 107300) is associated with type I antithrombin deficiency (ATD), that constitutes the most severe thrombophilia since it significantly increases the risk of venous thrombosis (OR:20-30). ${ }^{2}$ Routine investigation of ATD combines functional assays, antigen quantification and genetic analyses. Causal variants are identified in SERPINC1 for $70 \%$ of cases, whilst $5 \%$ of patients harbor defects in other genes and 25\% remain without a genetic diagnosis. $^{2}$ The majority of reported pathogenic variants in SERPINC1 are small genetic defects $(63 \%$ single-nucleotide variants and $28 \%$ indels), with structural variants (SVs) accounting for a smaller proportion of cases. ${ }^{3 ; 4}$

Structural variants are genomic rearrangements involving more than 50 nucleotides that contribute to genomic diversity and function, evolution, and can cause somatic and germline diseases. ${ }^{5-7}$ Despite improvements in genomic technologies, characterization of SVs remains challenging and the full spectrum of SVs is not achieved by routine methods such as microarrays or other targeted sequencing approaches. In ATD, the detection and characterization of SVs remain particularly challenging due to the high number of repetitive elements in and around SERPINC1 $35 \%$ of SERPINC1 sequence are interspersed repeats). ${ }^{8}$ Copy number variants causing ATD are routinely identified in specialized centers by multiplex ligation-dependent probe amplification (MLPA), ${ }^{2}$ but this technology does not consider the full spectrum of SVs. Additionally, it does not provide a nucleotide-level resolution, which is important for confirming causality and reveal insights into SVs formation..$^{9-11}$ These limitations may now be addressed by long reads, that can span repetitive or other problematic regions, allowing identification and characterization of SVs. ${ }^{10 ; 12-16}$

Here, we report on the results of long-read wholegenome sequencing (LR-WGS) on 19 unrelated cases with ATD, where routine molecular tests were either negative, ambiguous, or did not fully characterize a $\mathrm{SV}$, in order to identify, resolve and investigate the most likely molecular mechanism of formation of causal SVs involved in this severe thrombophilia.

Nineteen unrelated individuals with ATD were selected from our cohort of 340 cases, recruited between 1994 and 2019: 8 patients with causal SVs identified by MLPA were included for variant characterization and investigation of the potential mechanisms of formation, and 11 patients were selected because multiple independent genetic studies evaluating SERPINC1 had failed to identify causal variants (Table S1, Supplementary Methods). Measurement of antithrombin levels and function were performed for all participants as previously described. 17; 18 LR-WGS was performed using the PromethION platform (Oxford Nanopore Technologies) and a multi-modal analysis workflow for the sensitive detection of SVs was developed (http://github.com/who-blackbird/magpie) and applied (Figure 1A, Supplemental Methods). Detailed information is provided in Supplemental Methods.

Nanopore sequencing in 21 runs produced reads with an average length of $4,499 \mathrm{bp}$ and a median genome coverage of $16 x$ (Figure 1A-B). After a detailed quality control analysis (Figure S1) 83,486 SVs were identified, consistent with previous reports using LRWGS (Figure S2)..$^{12}$ Focusing on rare variants (allele count $<=10$ in gnomAD v3, NIHR BioResource and NGC project) $15 ; 19 ; 20$ in SERPINC1 and flanking regions, 10 candidate heterozygous SVs were observed in 9 individuals (Figure 1C). Visual inspection of read alignments identified an additional heterozygous $\mathrm{SV}$ in a region of low coverage involving SERPINC1.

First, Nanopore sequencing resolved the precise configuration of SVs previously identified by MLPA in 8 individuals (P1-P8). SVs were identified independently of their size (from $7 \mathrm{~Kb}$ to $968 \mathrm{~Kb}$, restricted to SERPINC1 or involving neighboring genes) and their type (six deletions, one tandem duplication and one complex SV) (Figure 2, Table S1). Importantly, SVs for two cases with previous inconsistent or ambiguous results were characterized by Nanopore sequencing (P2 and P6) (Figure 2, Table S1).

For the first case (P2), MLPA detected a deletion of exon 1, but long-range PCR followed by NGS suggested a deletion of exons 1 and 2 . The discordant results were explained by a complex SV in SERPINC1 revealed by Nanopore sequencing, that resulted in a dispersed duplication of exon 3 and the deletion of exon 1, both in the same allele (Figure S3A). Although complex SVs have already been associated with human disease, ${ }^{10 ; 21}$ this is the first report of a germline complex rearrangement involved in ATD, that was 


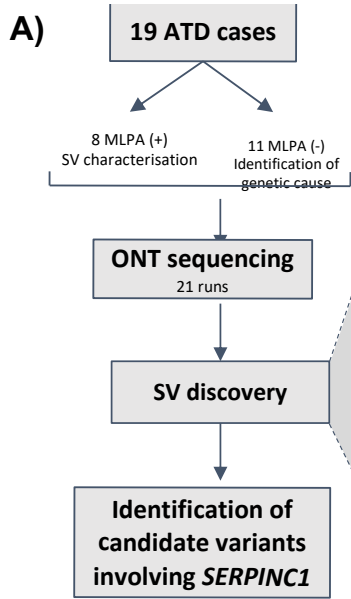

B)

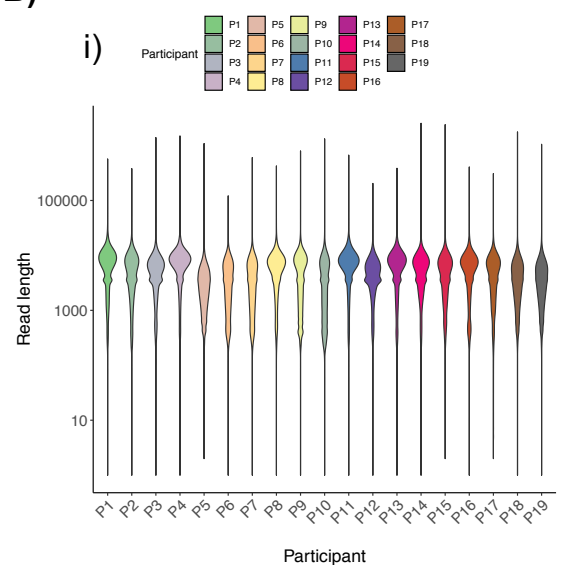

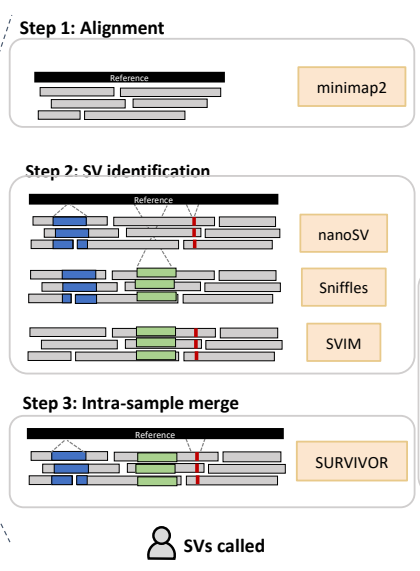

8 svs called

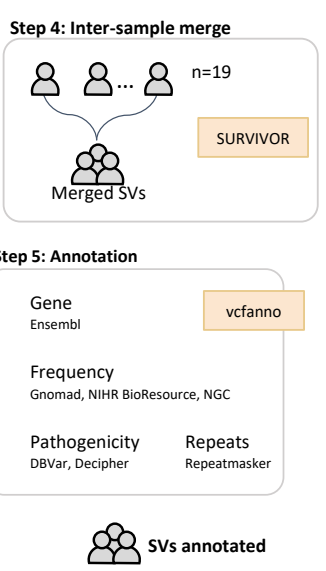

C)

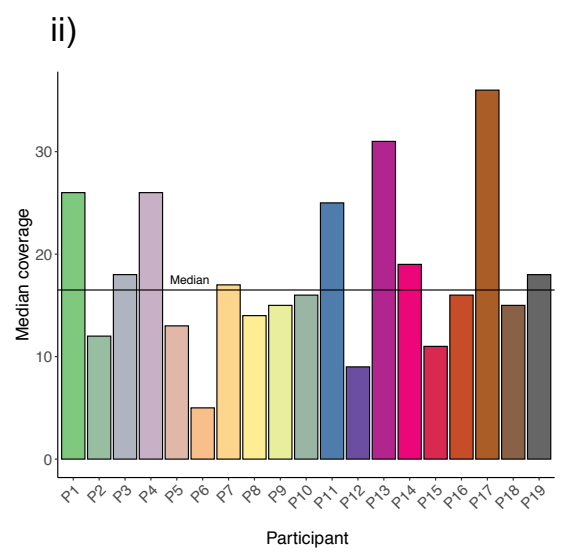

D)

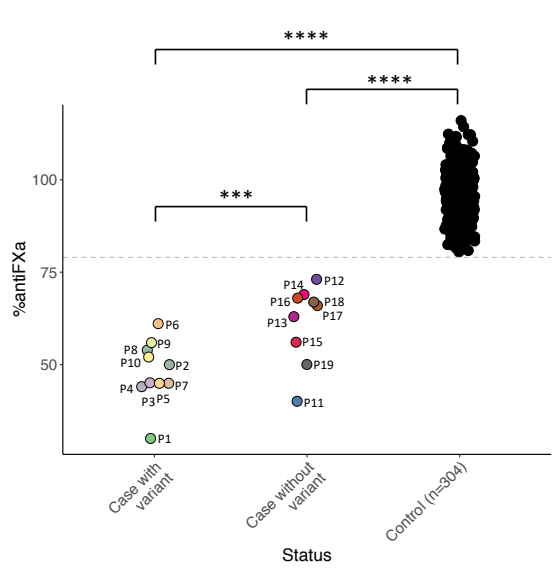

Figure 1. Long-read sequencing workflow and results. (A) Overview of the general stages of the SVs discovery workflow. Algorithms used are depicted in yellow boxes. (B) Nanopore sequencing results. i) Sequence length template distribution. Average read length was 4,499 bp ( $s d \pm 4,268$ ); the maximum read length observed was 2.5Mb. ii) Genome median coverage per participant. The average across all samples was $16 x$ (sd \pm 7.7$)$. (C) Filtering approach and number of SVs obtained per step. SERPINC1 + promoter region corresponds to [GRCh38/hg38] Chr1:173,903,500-173,931,500. (D) anti-FXa percentage levels for the participants with a variant identified (P1-P10), cases without a candidate variant (P11-P19) and 300 controls from our internal database. The statistical significance is denoted by asterisks $\left({ }^{*}\right)$, where ${ }^{* * *} P<0.001,{ }^{* * * *} P \leq 0.0001$. $p$-values calculated by oneway ANOVA with Tukey's post-hoc test for repeated measures. ATD=Antithrombin Deficiency; ONT=Oxford Nanopore Technologies; SV=Structural Variant.

also confirmed by Sanger sequencing in the affected daughter of P2. Further investigations would be required to elucidate whether the complex SV was formed by one or two independent mutational events.

For the second case (P6), MLPA detected a duplication of exons 1, 2 and 4 and a deletion of exon 6 . Here, our sequencing approach identified a tandem duplication of exons 1 to 5 , which was confirmed by long-range PCR (Figure S3B) and observed to be present in the affected son of P6.
Then, we aimed to identify new disease-causing variants in the remaining 11 participants. Remarkably, two cases (P9 and $\mathrm{P} 10$ ) presented an insertion of a SINE-VNTR-Alu (SVA) retroelement of $2,440 \mathrm{bp}$ (Figure 2, Table S1), suspected to induce transcriptional interference of SERPINC1. De novo assembly using the sequencing data of $P 9$ revealed an antisense-oriented SVA element flanked by a target site duplication (TSD) of 14 bp (Figure 2C), consistent with a target-primed reverse transcription mechanism of insertion into the genome. ${ }^{1 ;} 22$ Interestingly, the TSD in both individuals was the same, suggesting a shared mechanism of formation or 


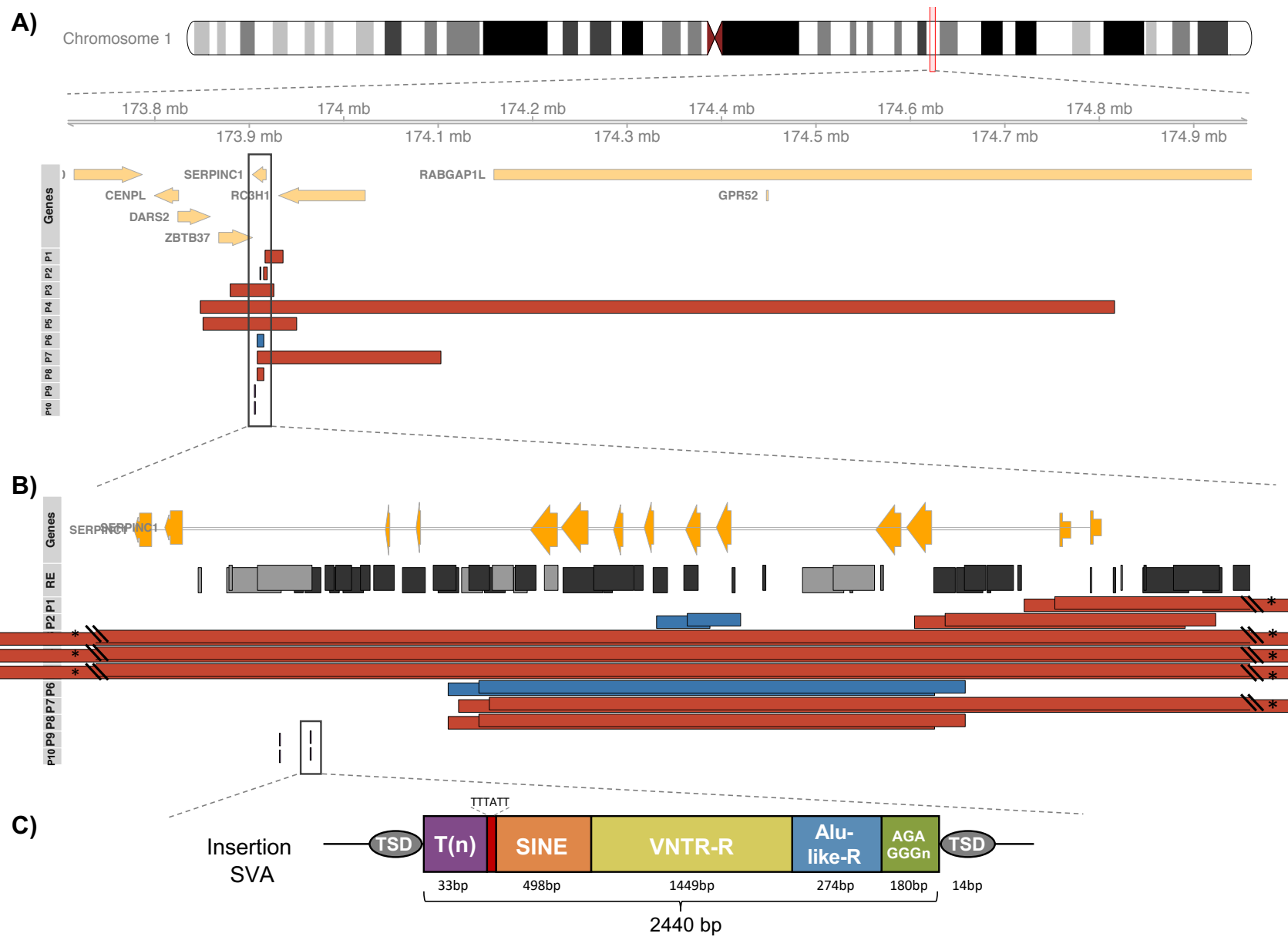

Figure 2. Candidate SVs identified by long-read sequencing. (A) Schematic of chromosome 1 followed by protein coding genes falling in the zoomed region (1q25.1). SVs for each participant $(P)$ are colored in red (deletions) and blue (duplications). The insertion identified in $P 9$ and $P 10$ is shown with a black line. (B) Schematic of SERPINC1 gene (NM_000488) followed by repetitive elements (RE) in the region. SINEs and LINEs are colored in light and dark grey respectively. Asterisks are present where the corresponding breakpoint falls within a RE. (C) Characteristics of the antisense-oriented SVA retroelement (respect to the canonical sequence) ${ }^{1}$ observed in P9. Length of the fragments are subject to errors from nanopore sequencing. TSD=Target site duplication.

a founder effect. The inserted sequence was aligned to the canonical SVA A-F sequences (Figure S4A) and it was observed to be closest to the SVA E in the phylogenetic tree (Figure S4B). Moreover, the VNTR sub-element harbored $1,449 \mathrm{bp}$, which was longer than the typical $\sim 520 \mathrm{bp}$-long VNTR in the canonical sequences.

These results highlight the heterogeneous genomic landscape of SVA sequences and underscore the importance of their characterization in order to obtain a reliable catalogue of novel mobile elements to identify and interpret this type of causal variants in other patients and other disorders where

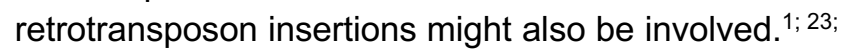
24 This characterization has been historically challenging by the application of classic technologies, but here we show that it can be achieved by de novo assembly of long-reads. The SVA insertion was confirmed in P9, P10 and two other affected relatives by specifically designed PCR amplification and Sanger sequencing facilitated by the Nanopore data (Figure S5). SVA retroelements are challenging to amplify given their genomic characteristics (GC-rich sequences and length). Here, multiple PCRs were attempted, and the final amplified product was only obtained by using an internal SVA primer (Figure S5).

Finally, breakpoint analysis was performed to investigate the mechanism underlying the formation of these SVs involving SERPINC1. Nanopore sequencing facilitated primer design to perform 
Sanger sequencing confirmations for all the new formed junctions, demonstrating a $100 \%$ accuracy in $7 / 10(70 \%)$ SVs called. Repetitive elements (RE) were detected in all the SVs, with Alu elements being the most frequent (16/24, 67\%) (Table S2). Alu-mediated SVs have been previously reported as associated with ATD, ${ }^{25}$ and their frequency is consistent with the high proportion of Alu sequences in SERPINC1 (22\% of intronic sequence) ${ }^{26}$ Additionally, breakpoint analysis identified microhomologies $(7 / 11,64 \%)$ and insertions, deletions or duplications $(7 / 11,64 \%)$ (Figure S6).

Specific mutational signatures can yield insights into the mechanisms by which the SVs are formed. Our results suggest a replication-based mechanism (such as BIR/MMBIR/FoSTeS) for most of the cases (P1P8). ${ }^{27}$ Importantly, we observed a non-random formation driven by the presence of REs in some of the SVs. For example, an Alu element in intron 1 was involved in the SVs of P6 and P8, and an Alu element in intron 5 was involved in SVs of P6, P7 and P8 (Figure 2B, Table S3). It has been suggested that RE may provide larger tracks of microhomologies, also termed 'microhomology islands', that could assist strand transfer or stimulate template switching during repair by a replication-based mechanism. ${ }^{27}$ These microhomology islands were present in the SVs of 4 cases (P4, P6-P8), highlighting the important role that RE play in the formation of non-recurrent, but nonrandom, SVs.

Overall, we resolved SVs affecting SERPINC1 in 10 individuals with ATD. However, 9 additional cases remain as yet unresolved, three of whom reported to have familial disease. An explanation may be that the causal variant was missed due to low coverage, or alternatively the variant is located in an unidentified transacting gene or in a regulatory element for SERPINC1, as we have recently reported for other genes. ${ }^{15}$ The observation that the ATD patients without causal SVs have significantly higher anti-FXa activity than those with SVs (Figure 1D) is supportive of the notion that causal variants may regulate gene expression.

Here, we show how LR-WGS can be used to resolve SVs causal of ATD, independently of the length or the type, that can be missed, misunderstood or misclassified by routine molecular diagnostic methods. Moreover, we report for the first time a germline complex rearrangement and the insertion of a SVA retroelement as the genetic defect responsible of ATD and reveal insights into the mechanisms of formation of these SVs. Altogether this study highlights the importance of identifying a new class of causal variants to improve diagnostic rates, to provide accurate family counselling and to facilitate decision making about long-term thromboprophylaxis.

\section{Supplemental data}

Supplemental methods, figures and tables are provided in separate documents, which will be linked directly from bioRxiv.

\section{Declaration of Interests}

The authors declare that they have no conflicts of interest.

\section{Acknowledgments}

We thank the participants involved in this study and their families. We thank NIHR BioResource volunteers for their participation, and gratefully acknowledge NIHR BioResource centers, NHS Trusts and staff for their contribution. We thank the National Institute for Health Research, NHS Blood and Transplant, and Health Data Research UK as part of the Digital Innovation Hub Programme. The views expressed are those of the author(s) and not necessarily those of the $\mathrm{NHS}$, the NIHR or the Department of Health and Social Care. This work was supported by the National Institute for Health Research England (NIHR) for the NIHR BioResource project (grant numbers RG65966 and RG94028), the PI18/00598 project (ISCIII $y$ FEDER) and the 19873/GERM/15 project (Fundación Séneca). All participants provided written informed consent to participate in the study. The study was approved by the East of England Cambridge South national institutional review board (13/EE/0325). The research conforms to the principles of the Declaration of Helsinki.

\section{Data and Code Availability}

Sequence data for all the individuals in this work have been deposited at the European Genome-Phenome Archive under the accession number EGAD00001006254. The workflow developed for the 
detection of SVs is publicly available at http://github.com/who-blackbird/magpie.

\section{Authorship Contributions}

BMB, WHO, JC and ASJ designed the study. MMB, LS, JP, AM, NG, FLR and VV helped with study design. $\mathrm{BMB}, \mathrm{MMB}, \mathrm{JP}, \mathrm{AM}$ performed laboratory experiments and analyzed the experimental data.

JS performed sample preparation and executed longread sequencing. ASJ developed the analysis workflow for long-read sequencing, applied this to data processing and performed the computational and statistical analyses. BMB performed computational analyses and variant validation. JM, FV, provided valuable insight into CGHa and NGS data analysis. $\mathrm{AU}, \mathrm{MF}, \mathrm{MP}$ and PM recruited participants and collected the clinical data and samples. BMB, WHO, $\mathrm{JC}$ and $\mathrm{ASJ}$ wrote the manuscript. All authors read and approved the final manuscript.

\section{References}

1. Payer, L.M., and Burns, K.H. (2019). Transposable elements in human genetic disease. Nat Rev Genet 20, 760-772.

2. Corral, J., de la Morena-Barrio, M.E., and Vicente, V. (2018). The genetics of antithrombin. Thromb Res 169, 23-29.

3. Stenson, P.D., Ball, E.V., Howells, K., Phillips, A.D., Mort, M., and Cooper, D.N. (2009). The Human Gene Mutation Database: providing a comprehensive central mutation database for molecular diagnostics and personalised genomics. Human Genomics 4, 69.

4. Beauchamp, N.J., Makris, M., Preston, F.E., Peake, I.R., and Daly, M.E. (2000). Major structural defects in the antithrombin gene in four families with type I antithrombin deficiency-partial/complete deletions and rearrangement of the antithrombin gene. Thromb Haemost 83, 715721.

5. Sudmant, P.H., Rausch, T., Gardner, E.J., Handsaker, R.E., Abyzov, A., Huddleston, J., Zhang, Y., Ye, K., Jun, G., Fritz, M.H.-Y., et al. (2015). An integrated map of structural variation in 2,504 human genomes. Nature 526, 75-81.

6. Stankiewicz, P., and Lupski, J.R. (2010). Structural variation in the human genome and its role in disease. Annu Rev Med 61, 437-455.

7. Collins, R.L., Brand, H., Karczewski, K.J., Zhao, X., Alfoldi, J., Francioli, L.C., Khera, A.V.,
Lowther, C., Gauthier, L.D., Wang, H., et al. (2020). A structural variation reference for medical and population genetics. Nature 581, 444-451.

8. de Koning, A.P.J., Gu, W., Castoe, T.A., Batzer, M.A., and Pollock, D.D. (2011). Repetitive elements may comprise over two-thirds of the human genome. PLoS Genet 7, e1002384.

9. Ordulu, Z., Kammin, T., Brand, H., Pillalamarri, V., Redin, C.E., Collins, R.L., Blumenthal, I., Hanscom, C., Pereira, S., Bradley, I., et al. (2016). Structural Chromosomal Rearrangements Require Nucleotide-Level Resolution: Lessons from Next-Generation Sequencing in Prenatal Diagnosis. Am J Hum Genet 99, 1015-1033.

10. Sanchis-Juan, A., Stephens, J., French, C.E., Gleadall, N., Mégy, K., Penkett, C., Shamardina, O., Stirrups, K., Delon, I., Dewhurst, E., et al. (2018). Complex structural variants in Mendelian disorders: identification and breakpoint resolution using short- and long-read genome sequencing. Genome Med 10, 95.

11. Lam, H.Y., Mu, X.J., Stutz, A.M., Tanzer, A., Cayting, P.D., Snyder, M., Kim, P.M., Korbel, J.O., and Gerstein, M.B. (2010). Nucleotideresolution analysis of structural variants using BreakSeq and a breakpoint library. Nat Biotechnol 28, 47-55.

12. Beyter, D., Ingimundardottir, H., Eggertsson, H.P., Bjornsson, E., Kristmundsdottir, S., Mehringer, S., Jonsson, H., Hardarson, M.T., Magnusdottir, D.N., Kristjansson, R.P., et al. Long read sequencing of 1,817 Icelanders provides insight into the role of structural variants in human disease.

13. Sedlazeck, F.J., Rescheneder, P., Smolka, M., Fang, H., Nattestad, M., von Haeseler, A., and Schatz, M.C. (2018). Accurate detection of complex structural variations using singlemolecule sequencing. Nat Methods 15, 461-468. 14. Cretu Stancu, M., van Roosmalen, M.J., Renkens, I., Nieboer, M.M., Middelkamp, S., de Ligt, J., Pregno, G., Giachino, D., Mandrile, G., Espejo Valle-Inclan, J., et al. (2017). Mapping and phasing of structural variation in patient genomes using nanopore sequencing. Nat Commun 8, 1326.

15. Turro, E., Astle, W.J., Megy, K., Graf, S., Greene, D., Shamardina, O., Allen, H.L., Sanchis-Juan, A., Frontini, M., Thys, C., et al. (2020). Whole-genome sequencing of patients with rare diseases in a national health system. Nature. 
16. De Coster, W., De Rijk, P., De Roeck, A., De Pooter, T., D'Hert, S., Strazisar, M., Sleegers, K., and Van Broeckhoven, C. (2019). Structural variants identified by Oxford Nanopore PromethION sequencing of the human genome. Genome Res 29, 1178-1187.

17. Morena-Barrio, M.d.I., de la Morena-Barrio, M., Sandoval, E., Llamas, P., Wypasek, E., Toderici, M., Navarro-Fernández, J., RodríguezAlen, A., Revilla, N., López-Gálvez, R., et al. (2017). High levels of latent antithrombin in plasma from patients with antithrombin deficiency. Thrombosis and Haemostasis 117, 880-888.

18. de la Morena-Barrio, M.E., MartínezMartínez, I., de Cos, C., Wypasek, E., Roldán, V., Undas, A., van Scherpenzeel, M., Lefeber, D.J., Toderici, M., Sevivas, T., et al. (2016). Hypoglycosylation is a common finding in antithrombin deficiency in the absence of a SERPINC1 gene defect. J Thromb Haemost 14, 1549-1560.

19. French, C.E., Delon, I., Dolling, H., SanchisJuan, A., Shamardina, O., Megy, K., Abbs, S., Austin, T., Bowdin, S., Branco, R.G., et al. (2019). Whole genome sequencing reveals that genetic conditions are frequent in intensively ill children. Intensive Care Med 45, 627-636.

20. Karczewski, K.J., Francioli, L.C., Tiao, G., Cummings, B.B., Alfoldi, J., Wang, Q., Collins, R.L., Laricchia, K.M., Ganna, A., Birnbaum, D.P., et al. (2020). The mutational constraint spectrum quantified from variation in 141,456 humans. Nature 581, 434-443.

21. Collins, R.L., Brand, H., Redin, C.E., Hanscom, C., Antolik, C., Stone, M.R., Glessner, J.T., Mason, T., Pregno, G., Dorrani, N., et al. (2017). Defining the diverse spectrum of inversions, complex structural variation, and chromothripsis in the morbid human genome. Genome Biol 18, 36.

22. Vogt, J., Bengesser, K., Claes, K.B.M., Wimmer, K., Mautner, V.-F., van Minkelen, R., Legius, E., Brems, H., Upadhyaya, M., Högel, J., et al. (2014). SVA retrotransposon insertionassociated deletion represents a novel mutational mechanism underlying large genomic copy number changes with non-recurrent breakpoints. Genome Biol 15, R80.

23. Hancks, D.C., and Kazazian, H.H., Jr. (2016). Roles for retrotransposon insertions in human disease. Mob DNA 7, 9.

24. Kazazian, H.H., Jr., and Moran, J.V. (2017). Mobile DNA in Health and Disease. N Engl J Med 377, 361-370.
25. Picard, V., Chen, J.-M., Tardy, B., Aillaud, M.F., Boiteux-Vergnes, C., Dreyfus, M., Emmerich, J., Lavenu-Bombled, C., Nowak-Göttl, U., Trillot, N., et al. (2010). Detection and characterisation of large SERPINC1 deletions in type I inherited antithrombin deficiency. Hum Genet 127, 45-53. 26. Olds, R.J., Lane, D.A., Chowdhury, V., De Stefano, V., Leone, G., and Thein, S.L. (1993). Complete nucleotide sequence of the antithrombin gene: evidence for homologous recombination causing thrombophilia. Biochemistry 32, 4216-4224.

27. Carvalho, C.M.B., and Lupski, J.R. (2016). Mechanisms underlying structural variant formation in genomic disorders. Nat Rev Genet $17,224-238$. 